\title{
Correction: Non-GVHD ocular complications after hematopoietic cell transplantation: expert review from the Late Effects and Quality of Life Working Committee of the CIBMTR and Transplant Complications Working Party of the EBMT
}

Yoshihiro Inamoto ${ }^{1} \cdot$ Igor Petriček $^{2} \cdot$ Linda Burns $^{3} \cdot$ Saurabh Chhabra $^{4} \cdot$ Zack DeFilipp $^{5} \cdot$ Peiman Hematti $^{6}$. Alicia Rovó ${ }^{7} \cdot$ Raquel Schears $^{8} \cdot$ Ami Shah $^{9} \cdot$ Vaibhav Agrawal $^{10} \cdot$ Aisha Al-Khinji $^{11} \cdot$ Ibrahim Ahmed $^{12} \cdot$ Asim Ali $^{13}$. Mahmoud Aljurf ${ }^{14} \cdot$ Hassan Alkhateeb $^{8}$ - Amer Beitinjaneh ${ }^{15} \cdot$ Neel Bhatt $^{4}$ - Dave Buchbinder ${ }^{15}$ - Michael Byrne ${ }^{16}$.

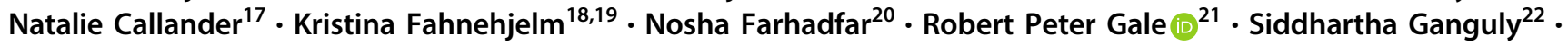
Gerhard C. Hildebrandt ${ }^{23}$ - Erich Horn ${ }^{20}$. Ann Jakubowski ${ }^{24} \cdot$ Rammurti T. Kamble $^{25}$ • Jason Law ${ }^{26}$ - Catherine Lee ${ }^{27}$. Sunita Nathan ${ }^{28}$ - Olaf Penack ${ }^{29} \cdot$ Ravi Pingali $^{30} \cdot$ Pinki Prasad $^{31} \cdot$ Drazen Pulanic $^{32,33,34} \cdot$ Seth Rotz ${ }^{35}$. Aditya Shreenivas $^{36}$ - Amir Steinberg ${ }^{36} \cdot$ Khalid Tabbara $^{37}$. André Tichelli $^{38} \cdot$ Baldeep Wirk $^{39} \cdot$ Jean Yared $^{40}$. Grzegorz W. Basak $\mathbb{C}^{41} \cdot$ Minoo Battiwalla $^{42} \cdot$ Rafael Duarte $^{43} \cdot$ Bipin N. Savani ${ }^{44} \cdot$ Mary E. D. Flowers $^{45} \cdot$ Bronwen E. Shaw ${ }^{46} \cdot$ Nuria Valdés-Sanz ${ }^{47}$

Published online: 27 February 2019

C Springer Nature Limited 2019

Correction to: Bone Marrow Transplant; 2018 https://doi.org/10.1038/s41409-018-0339-6; published online 7 Dec 2018.
In the original version of this article, author 'Aisha AlKhinji' was incorrectly listed as 'Aisha Ahmed'. This has now been corrected in both the PDF and HTML versions of the article to 'Aisha Al-Khinji'.

These authors contributed equally: Yoshihiro Inamoto, Igor Petriček, Nuria Valdés-Sanz

The original article can be found online at https://doi.org/10.1038/ s41409-018-0339-6.

Yoshihiro Inamoto

yinamoto@ncc.go.jp

Extended author information available on the last page of the article 
1 Department of Hematopoietic Stem Cell Transplantation, National Cancer Center Hospital, Tokyo, Japan

2 Department of Ophthalmology, University Clinical Hospital Zagreb, Zagreb, Croatia

3 CIBMTR(Center for International Blood and Marrow Transplant Research), National Marrow Donor Program/Be The Match, Minneapolis, MN, USA

4 Medical College of Wisconsin, Milwaukee, WI, USA

5 Blood and Marrow Transplant Program, Massachusetts General Hospital, Boston, MA, USA

6 Division of Hematology/Oncology/Bone Marrow Transplantation, Department of Medicine, University of Wisconsin Hospital and Clinics, Madison, WI, USA

7 Universitatsspital Bern, Bern, Switzerland

8 Mayo Clinic Rochester, Rochester, MN, USA

9 Division of Stem Cell Transplantation and Regenerative Medicine, Lucille Packard Children's Hospital, Stanford School of Medicine, Palo Alto, CA, USA

10 Indiana University Simon Cancer Center, Indianapolis, IN, USA

11 University of California, San Francisco, CA, USA

12 Department of Hematology Oncology and Bone Marrow Transplantation, The Children's Mercy Hospitals and Clinics, Kansas City, MO, USA

13 St. Louis Medical Clinic, St. Louis, MO, USA

14 Department of Oncology, King Faisal Specialist Hospital Center and Research, Riyadh, Saudi Arabia

15 Division of Pediatrics Hematology, Children's Hospital of Orange County, Orange, CA, USA

16 Vanderbilt University Medical Center, Nashville, TN, USA

17 University of Wisconsin Carbone Cancer Center, Madison, WI, USA

18 Department of Clinical Neuroscience, Karolinska Institutet, St. Erik Eye Hospital, Stockholm, Sweden

19 Department of Clinical Neuroscience, Institute of Neuroscience and Physiology, Sahlgrenska Academy, University of Gothenburg, Gothenburg, Sweden

20 Shands Healthcare and University of Florida, Gainsville, FL, USA

21 Hematology Research Centre, Division of Experimental Medicine, Department of Medicine, Imperial College London, London, United Kingdom

22 Division of Hematological Malignancy and Cellular Therapeutics, University of Kansas Health System, Kansas City, KS, USA

23 Markey Cancer Center, University of Kentucky, Lexington, KY, USA
24 Division of Hematologic Oncology, Memorial Sloan Kettering Cancer Center, New York, NY, USA

25 Division of Hematology and Oncology, Center for Cell and Gene Therapy, Baylor College of Medicine, Houston, TX, USA

26 Tufts Medical Center, Boston, MA, USA

27 Utah Blood and Marrow Transplant Program Huntsman Cancer Institute, University of Utah, Salt Lake City, UT, USA

28 Rush University Medical Center, Chicago, IL, USA

29 Division of Haematology and Oncology, Department of Internal Medicine, Charité University Medicine, Campus Rudolf Virchow, Berlin, Germany

30 Houston Methodist Hospital, Houston, TX, USA

31 Children's Hospital, New Orleans, LA, USA

32 Division of Hematology, Department of Internal Medicine, University Hospital Center Zagreb, Zagreb, Croatia

33 School of Medicine, University of Zagreb, Zagreb, Croatia

34 Faculty of Medicine Osijek, J. J. Strossmayer University of Osijek, Osijek, Croatia

35 Cincinnati Children's Hospital Medical Center, Cincinnati, OH, USA

36 Division of Hematology and Oncology, Mount Sinai Hospital, New York, NY, USA

37 The School of Medicine, The Wilmer Ophthalmological Institute of The Johns Hopkins University, Baltimore, MD, USA

38 University Hospital Basel, Basel, Switzerland

39 Division of Bone Marrow Transplant, Seattle Cancer Care Alliance, Seattle, WA, USA

40 Blood and Marrow Transplantation Program, Division of Hematology/Oncology, Department of Medicine, Greenebaum Cancer Center, University of Maryland, Baltimore, MD, USA

41 Medical University of Warsaw, Warsaw, Poland

42 Hematology Branch, Sarah Cannon BMT Program, Nashville, TN, USA

43 University Hospital Puerta de Hierro, Madrid, Spain

44 Division of Hematology/Oncology, Department of Medicine, Vanderbilt University Medical Center, Nashville, TN, USA

45 Clinical Research Division, Fred Hutchinson Cancer Research Center, Seattle, WA, USA

46 CIBMTR (Center for International Blood and Marrow Transplant Program), Department of Medicine, Medical College of Wisconsin, Milwaukee, WI, USA

47 Department of Ophthalmology, Hospital Universitario Puerta de Hierro, Madrid, Spain 